Forest habitat connectivity of the natural protected areas for the white tailed deer (Odocoileus virginianus) in Jalisco State, Mexico

\title{
Conectividad del hábitat forestal de las áreas protegidas para el venado cola blanca (Odocoileus virginianus) en el estado de Jalisco, México
}

\author{
Raymundo Villavicencio García ${ }^{1 *}$, Rosaura Avila Coria ${ }^{1}$, \\ Sergio Guerrero Vázquez², Ana Luisa Santiago Pérez¹, \\ Eduardo Treviño Garza ${ }^{3}$
}

\section{Resumen}

Se utilizó el índice integral de conectividad (IIC) con el propósito de identificar aquellas coberturas forestales tanto críticas como prioritarias para el mantenimiento de la conectividad de hábitat forestal, en función a la distancia de desplazamiento del venado cola blanca. Se empleó información cartográfica de uso de suelo y vegetación del inventario nacional forestal para el estado de Jalisco de 2011 (serie V) del INEGI. Se calculó el rango de importancia relativa del Conjunto del Paisaje y la disponibilidad de hábitat mediante las fracciones de IIC: intra que es la contribución del parche en términos del hábitat disponible en su interior, flux que corresponde al flujo de dispersión recibido $\mathrm{u}$ originado a través de las conexiones del parche con el resto, siendo este el origen o destino de dichas conexiones y flujos de dispersión y connector que evalúa la contribución del parche como elemento conector o parche puente entre el resto de los parches de hábitat del paisaje. Estos índices cuantificaron las diferentes maneras en las que un determinado elemento del paisaje puede contribuir a la conectividad global del paisaje. Se sugiere a nivel municipal, en términos de ordenamiento territorial, la restauración y fomento forestal a fin de mejorar la calidad del hábitat y conexión entre parches forestales. Con el propósito de mantener la conectividad del hábitat a

\footnotetext{
${ }^{1}$ Departamento de Producción Forestal, Centro Universitario de Ciencias Biológicas y Agropecuarias (CUCBA), Universidad de Guadalajara (UDG). Camino Ing. Ramón Padilla Sánchez No. 2100 Predio Las Agujas, Zapopan, Jalisco, México. Tel: 37771150, ext. 33157; email: raymundo.villavicencio@academicos.udg.mx.

${ }^{2}$ Departamento de Botánica y Zoología, Centro de Estudios en Zoología. CUCBA-UDG. Camino Ing. Ramón Padilla Sánchez No. 2100 Predio Las Agujas, Zapopan, Jalisco, México. Tel: 37771150.

${ }^{3}$ Facultad de Ciencias Forestales, Universidad Autónoma de Nuevo León. Carretera Nacional No.85 Km. 145. Linares Nuevo León, México
} 
mediano y largo plazo y asegurar el éxito dispersivo de la especie hacia otros espacios naturales, deben considerarse como prioritarios aquellos parches con valor muy bajo, bajo y medio del $\mathrm{dIIC}_{k}$.

Palabras clave: Parche, Distancia, Dispersión, Conexo.

\section{Abstract}

The integral index of connectivity (IIC) was used in order to identify those forest cover both critical and priority for maintaining forest habitat connectivity, in function of movement distance. A land use and vegetation of Jalisco map was used (serie V, INEGI). The relative importance range of the whole landscape and habitat availability was calculated by three fractions of IIC. Intra is the contribution in terms of available habitat inside the patch. Flux corresponds to the leakage flux received or originated through connections patch with the rest of them, being the origin or destination of these connections and flows of dispersion. Finally Connector evaluates the contribution of the patch as a bridge connecting element or as a stepping stone between the remaining habitat patches of the landscape. These indexes quantify the different ways in which a particular element of the landscape can contribute to the overall landscape connectivity. It is suggested to the municipal level in terms of land management, forest restoration and promotion in order to improve habitat quality and connection between forest patches. In order to maintain the habitat connectivity in a medium and long term and ensure the dispersive success of the species towards other natural spaces, it should be considered as priority those patches with very low, low and medium value of $d I I C_{k}$.

Keywords: Patch, Distance, Dispersion, Related.

\section{Introducción}

El venado cola blanca (Odocoileus virginianus) es una especie con importancia ecológica, económica y social, ya que puede ser considerada como una especie clave en el equilibrio ecológico de casi todos los ecosistemas, por el papel que desempeña como animal herbívoro y como presa (Chávez, 
2012). Esta especie ha sido afectada en mayor o menor medida por los efectos de la fragmentación forestal, la disminución, pérdida y conectividad de hábitat, no solo en su distribución, sino también en su densidad poblacional; además de la deforestación y sus efectos asociados junto con la caza de subsistencia y deportiva, representan los principales motivos por los cuales no solo ha reducido su distribución natural en México y Centroamérica, sino también, quizá muchas de las áreas originales de distribución ya no representan a las poblaciones originales (Medina et al., 2015; Ortiz et al., 2006; Weber, 2014).

Si bien, tales efectos conllevan a tener un mayor impacto sobre algunos grupos faunísticos, uno de estos es el de los grandes mamíferos, según la especie y lo extenso del territorio que ocupe para su subsistencia; hasta ahora, la Norma Oficial Mexicana 059 de la Secretaria de Medio Ambiente y Recursos Naturales (DOF, 2010), no cataloga al del venado cola blanca, como una especie en riesgo, tal como se encuentran sujetas otras subespecies endémicas de México, como el venado bura de Sheldon (Odocoileus hemionus subespecie sheldoni) y el venado bura de Isla Cedros (Odocoileus hemionus subespecie cerrosensis), que si se encuentran en las categorías en peligro de extinción y amenazada.

Las áreas naturales protegidas (ANP) en México constituyen la estrategia base para la conservación de los ecosistemas emblemáticos para, proteger su biodiversidad; no obstante, como cualquier otra zona natural, las áreas de sus alrededores e incluso las internas a las ANP, son dinámicas y presentan cambios estructurales a menor o mayor escala por diversos factores, como son: las plagas e incendios forestales, la tala ilícita, la ganadería, la cacería furtiva y el crecimiento urbano; por consecuencia, la fragmentación y pérdida de hábitats aumenta y la conectividad del hábitat forestal disminuye (Villavicencio et al., 2009). La conectividad biológica es primordial en la actual gestión territorial, que podría mitigar algunos efectos de la fragmentación; por tal motivo, el objetivo del presente es identificar la conectividad del hábitat forestal y priorizar aquellos elementos para su mantenimiento en función a la dispersión para el venado cola blanca (Odocoileus virginianus) que se considera como objeto de conservación para este trabajo. 


\section{Materiales y métodos}

El área de estudio comprendió el estado de Jalisco, se localiza en el occidente de México, entre las coordenadas $22^{\circ} 45^{\prime} 00^{\prime \prime}$ y $18^{\circ} 55^{\prime} 00^{\prime \prime}$ Latitud Norte y $101^{\circ} 28^{\prime} 00^{\prime \prime}$ y $105^{\circ} 42^{\prime} 00^{\prime \prime}$ Longitud Oeste, colinda al norte con los estados de Nayarit, Zacatecas y Aguascalientes, al este con Zacatecas, Guanajuato y Michoacán; al sur con el Océano Pacifico, Colima y Michoacán y, al oeste con el Océano Pacifico y Nayarit (SEMARNAT, 2014), además forma parte de las regiones de la Sierra Madre Occidental y el Eje Neovolcánico. Su superficie es de 78,588 km² y se encuentra dividido en 125 municipios (DGDM, 2017). El límite altitudinal varía de 0 a 4,260 msnm. Asimismo se consideraron de manera específica para este estudio veinte Áreas Naturales Protegidas (ANP) administradas por los gobiernos federal, estatal y municipal, que en su conjunto suman una extensión de 7,333.57 km² y corresponden a las Reservas de la Biosfera de Sierra de Manantlán y Chamela-Cuixmala; el Parque Nacional Nevado de Colima; las Áreas de Protección de Flora y Fauna La Primavera y Sierra de Quila; la Cuenca Alimentadora del Distrito Nacional de Riego 043; los Santuarios las Islas de la Bahía de Chamela, la Playa Mismaloya, la Playa Teopa, la Playa El Tecuán y la Playa Cuixmala.

La áreas protegidas de ámbito estatal son: la Sierra del Águila, el Cerro Viejo ChupinayaLos Sabinos, el Bosque Mesófilo Nevado de Colima y el Estero El Salado. Las áreas protegidas municipales corresponden a la Barranca del Río Santiago, la Campana-Colomos III, el Bosque Los Colomos, el Bosque El Nixticuil y las Piedras Bola (Zepeda, 2013) (Figura 1).

El estudio de la conectividad de hábitat se fundamentó sobre las coberturas forestales del estado de Jalisco; de acuerdo a la última edición cartográfica de uso de suelo y vegetación para México (INEGI, 2013), que corresponde a la serie V a escala 1:250,000. El conjunto de datos vectoriales de esta capa enlista hasta setenta y uno diferentes categorías de comunidades, entre las que destaca el bosque de encino, el bosque de pino-encino, el bosque de pino, la selva baja caducifolia y la selva mediana caducifolia.

Para este trabajo se partió de la base que, las coberturas forestales se conforman por uno o varios mosaicos de elementos, los cuales son caracterizados por la ecología del paisaje como "elementos de hábitat" o bien, "parches" (Chuvieco, 2010); de tal manera que se pueden diferenciar los siguientes: matrices, corredores, parches o parches puente. Para el estudio de la conectividad 
de hábitat forestal, todos los tipos de ecosistemas y sus diferentes variantes se unificaron para considerarse como una Cobertura forestal, por el contrario, el resto de las superficies dedicadas a otros usos de suelo se les denominó Cobertura no forestal (Figura 2); ambas coberturas se contextualizan finalmente como Conjunto del Paisaje.

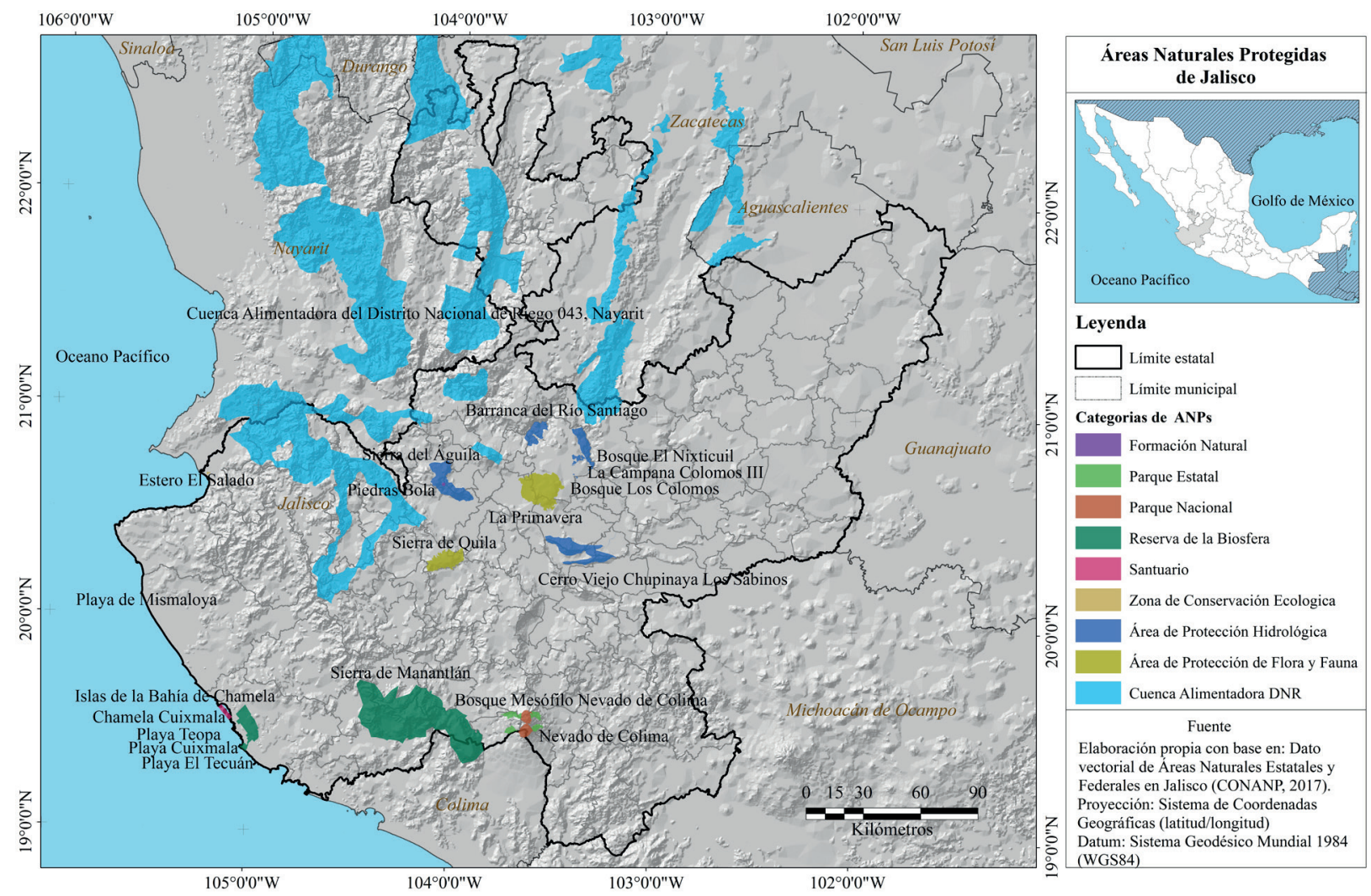

Figura 1. Localización del área de estudio (Estado de Jalisco) y ubicación de las áreas protegidas por categoría de manejo.

Para determinar el cálculo del valor de importancia relativa en la conectividad de cada elemento como enlace de hábitat incluidas las ANP en el Conjunto del Paisaje, se utilizó el Índice Integral de Conectividad (IIC) (Pascual-Hortal y Saura, 2006); su función se basa en el uso combinado de los Sistemas de Información Geográfica, estructuras de grafos y el valor de dispersión de la especie en estudio. El IIC se determinó utilizando los aspectos estructurales (distancia entre parches) y funcionales (área de cada parche) de la conectividad de hábitat que conforman el paisaje, así como la distancia mediana de desplazamiento de la especie en estudio. El valor del índice se obtiene de la siguiente formula: 


$$
I I C=\frac{\sum_{i=1}^{n} \sum_{j=1}^{n} \frac{a i \cdot a j}{1+n l_{i j}}}{A_{P}^{2}}
$$

Donde $a_{i}$ es el área de cada parche forestal, $A_{p}$ es el área total del Conjunto del Paisaje y $n l$ es el número de conexiones (entre parches) en el camino más corto (distancia topológica) entre los parches forestales $i$ y j. Para parches que no estén conectados (que pertenezcan a diferentes componentes o regiones conexas) el numerador de la suma de la ecuación es cero $\left(h_{i j}=\infty\right)$. Cuando $i=j$, entonces $n l_{i j}=0$ (no es necesario enlace alguno para alcanzar un parche desde si mismo) (Pascual-Hortal y Saura, 2006).

La importancia relativa de cada parche $\left(d I I C_{k}\right)$ para el mantenimiento de la conectividad se obtiene con la siguiente ecuación:

$$
d I I C_{k}=100 \cdot \frac{I I C-I I C_{e \lim , k}}{I I C}
$$

Donde $\mathrm{dIIC}_{k}$ es la importancia del elemento $k$ para el mantenimiento de la conectividad y disponibilidad de hábitat en el paisaje; el IIC figura como el valor del paisaje original (antes de la eliminación de algún elemento) y $I I C_{\text {elim, }}$ es el valor del índice detrás de la eliminación del elemento $k$. El cálculo de $d I I C_{k}$ para cada uno de los elementos del paisaje permite priorizar e identificar los elementos de hábitat más críticos para el mantenimiento de la conectividad; es decir, aquellos parches en los que la pérdida o deterioro del hábitat tendría un impacto más negativo sobre la conectividad del Conjunto del Paisaje. Dentro del mismo Conjunto del Paisaje, los diferentes parches tienen funciones diferentes que dependen de sus características intrínsecas y de su posición topológica dentro de una red de conectividad (De La Cruz y Maestre, 2013); de esta manera, el IIC se divide en tres fracciones que cuantifican las diferentes maneras en las que un elemento de hábitat $k$ en el Conjunto del Paisaje puede contribuir a la conectividad global del paisaje (De La Cruz y Maestre, 2013; Saura y Rubio, 2010). Por consiguiente, el valor de $d I I C_{k}$ se desglosa de acuerdo a la siguiente fórmula.

$$
d I I C_{k}=\text { dIICIntra }+ \text { dIICFlux } x_{k}+\text { dIICConnedor } r_{k}
$$

Donde el dIICIntra $a_{k}$ es la contribución del parche $k$ en términos del área (o calidad) de hábitat disponible en su interior (intrapatch connectivity). Esta fracción es independiente de la posición topológica de $k$ dentro del paisaje y la magnitud de las conexiones entre los diferentes parches, 
no depende de las capacidades de dispersión de las especies y tendría el mismo valor aunque $k$ estuviera completamente aislada. dIICFlux $x_{k}$ corresponde al flujo de dispersión (ponderado por el área $\mathrm{u}$ otro atributo utilizado para caracterizar los parches) recibido u originado a través de las conexiones del parche $k$ con el resto de los parches presentes en el paisaje, siendo $k$ el origen o destino de dichas conexiones y flujos de dispersión. Esta fracción depende del atributo del parche y de su posición en el paisaje con respecto al resto de los parches (interpatch connectivity). La fracción muestra lo bien conectado que está $k$ con el resto del hábitat existente en el paisaje, pero no la importancia de $k$ para mantener los flujos y conexiones entre otros elementos de hábitat (De La Cruz y Maestre, 2013).
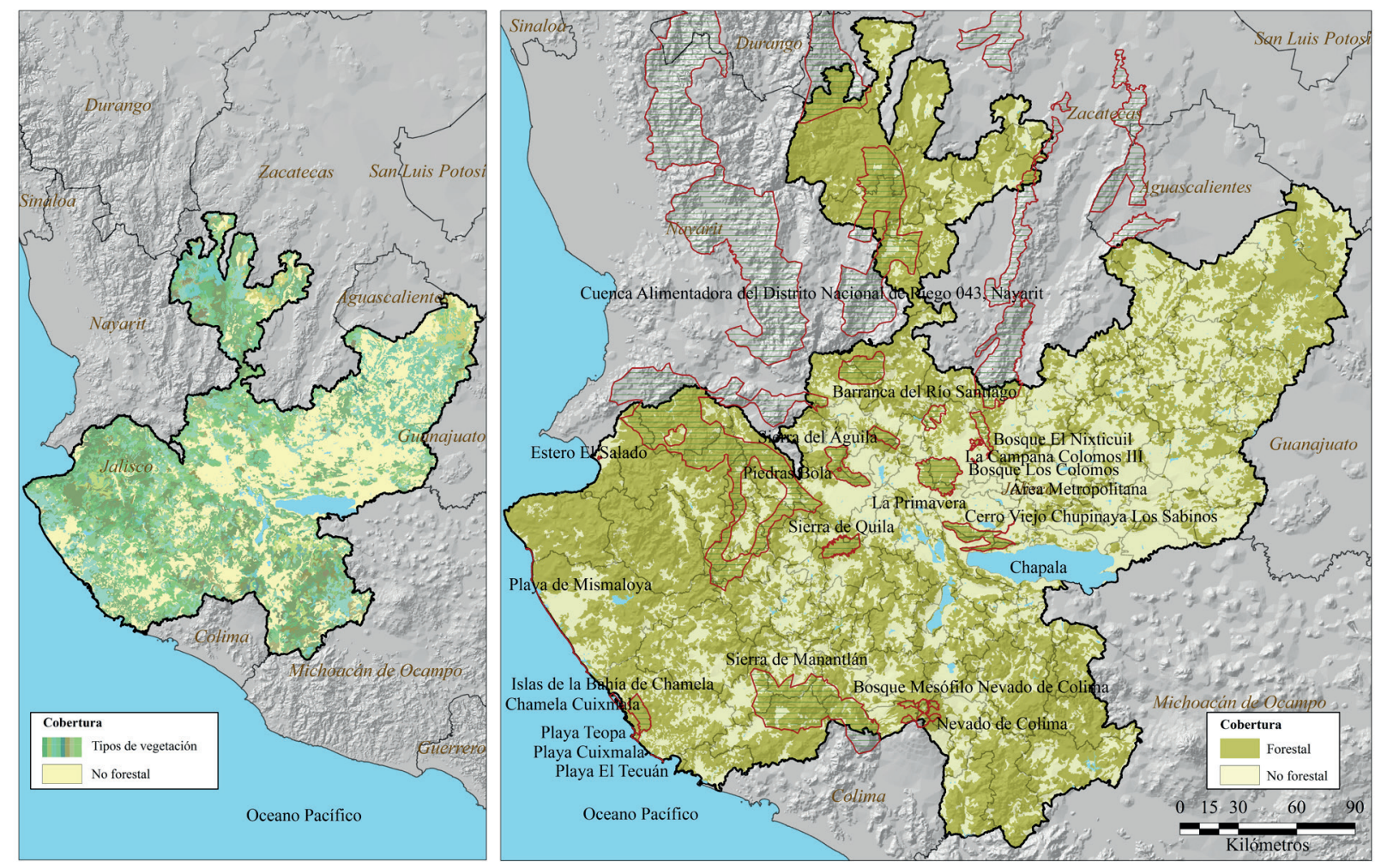

Figura 2. Coberturas de uso de suelo y vegetación (izq.) del estado de Jalisco y simplificación a Coberturas Forestales y no Forestales (der.).

dIICconnector ${ }_{k}$ evalúa la contribución del parche $k$ como elemento conector o parche puente entre el resto de los parches del paisaje. Es decir, mide hasta qué punto $k$ facilita los flujos dispersivos que no tienen su origen ni destino en $k$ pero que sí son potenciados y pasan a través de $k$. Esta fracción es independiente del área o cualquier otro atributo local del parche $k$ y solo depende de su posición topológica en el mosaico del paisaje (De La Cruz y Maestre, 2013). 
Para el cálculo de $d I I C_{k}$ y sus fracciones se utilizó el programa Conefor 2.6 (Saura y Torné, 2012).

La distancia mediana de desplazamiento del venado cola blanca, se determinó en 1.1 kilómetros, atribuido a la capacidad dispersiva referidos por: Bello et al., 2004; Ceballos y Oliva, 2005; Chávez, 2012; Fulbright y Ortega, 2007; Hernández, 2014; Ontiveros, 2012; SEMARNAT, 2014b; Weber, 2014y. La caracterización de cada elemento de hábitat o parche se definió en cinco categorías de importancia (muy alta, alta, media, baja y muy baja), estas se clasificaron en un Sistema de Información Geográfica mediante la opción clase de corte natural (Jenks), la cual realiza agrupaciones inherentes a los datos en función a la suma acumulada del valor relativo de $d I I C_{k}$ y sus respectivas fracciones. Finalmente el análisis es representado de manera cartográfica para identificar cada elemento de hábitat o parche crítico o prioritario para el mantenimiento de la conectividad.

\section{Resultados y Discusión}

De acuerdo al mapa de uso de suelo y vegetación del INEGI, la superficie forestal del estado de Jalisco es de 59.6\%, en este contexto, el Conjunto del Paisaje lo conforman diversos tipos de vegetación como, el bosque de encino y sus diferentes asociaciones con 14.1\%, el bosque de pino-encino y sus diferentes asociaciones con 9.9\%, el bosque de encino-pino y sus diferentes asociaciones con $4 \%$, el bosque de pino y sus diferentes asociaciones con $1.6 \%$, un uno por ciento más se suma de la comunidades como bosque de oyamel, bosque de táscate, bosque mesófilo de montaña y bosque de galería; por otro lado, la selva baja caducifolia y sus diferentes asociaciones ocupa el $18.7 \%$, seguido de la selva mediana subcaducifolia y sus diferentes asociaciones con $3.7 \%$.

Otras comunidades vegetales como el bosque de mezquite, el manglar, el matorral crasicaule, la vegetación sabanoide, la selva de galería, el palmar natural, el tular, la vegetación de dunas costeras y la vegetación halófila e hidrófila también ocurren en el estado de Jalisco, sin embargo, estas cubren menos de una unidad porcentual del territorio. Otras coberturas relevantes por su distribución dentro del Conjunto del Paisaje son las superficies agrícolas (30.8\%) y los pastizales inducidos (5.8\%) entre otros, los cuales suman el 40.3\% de las coberturas no forestal (Tabla I).

Dentro del Conjunto del Paisaje y sólo para el hábitat Cobertura forestal sin importar el tipo de vegetación que se represente, la Figura 3 muestra la distribución espacial de 548 parches forestales $\left(46,853.3 \mathrm{~km}^{2}\right)$ categorizados por el valor acumulado de $d I I C_{k}$ y con base a la distancia 
Tabla I. Tipo de categorías de comunidades de vegetación en el estado de Jalisco (Fuente: INEGI, 2013).

\begin{tabular}{|c|c|c|c|c|c|}
\hline Tipo de Cobertura & $\begin{array}{l}\text { Sup. } \\
\left(\mathbf{k m}^{2}\right)\end{array}$ & Sup. (\%) & Tipo de Cobertura & Sup. $\left(\mathbf{k m}^{2}\right)$ & Sup. $(\%)$ \\
\hline Bosque de encino & 5381.1 & 6.811 & Pastizal natural con veg. sec. arbustiva & 3383.2 & 4.282 \\
\hline Bosque de encino con veg. sec. arbórea & 644.7 & 0.816 & Pradera de alta montaña & 25.0 & 0.032 \\
\hline $\begin{array}{l}\text { Bosque de encino con veg. sec. } \\
\text { arbustiva }\end{array}$ & 5126.2 & 6.488 & Sabanoide & 127.1 & 0.161 \\
\hline Bosque de encino con veg. sec. halófila & 0.9 & 0.001 & Selva baja caducifolia & 4099.0 & 5.188 \\
\hline Bosque de encino pino & 2030.7 & 2.570 & $\begin{array}{l}\text { Selva baja caducifolia con veg. sec. } \\
\text { arbórea }\end{array}$ & 1169.4 & 1.480 \\
\hline $\begin{array}{l}\text { Bosque de encino pino con veg. sec. } \\
\text { arbórea }\end{array}$ & 267.3 & 0.338 & $\begin{array}{l}\text { Selva baja caducifolia con veg. sec. } \\
\text { arbustiva }\end{array}$ & 9481.8 & 12.002 \\
\hline $\begin{array}{l}\text { Bosque de encino pino con veg. sec. } \\
\text { arbustiva }\end{array}$ & 881.7 & 1.116 & $\begin{array}{l}\text { Selva baja caducifolia con veg. sec. } \\
\text { halófila }\end{array}$ & 1.3 & 0.002 \\
\hline Bosque de galería & 1.6 & 0.002 & $\begin{array}{l}\text { Selva baja espinosa caducifolia con veg. } \\
\text { sec. arbustiva }\end{array}$ & 40.7 & 0.052 \\
\hline $\begin{array}{l}\text { Bosque de galería con veg. sec. } \\
\text { arbórea }\end{array}$ & 1.8 & 0.002 & Selva baja subcaducifolia & 1.3 & 0.002 \\
\hline Bosque de mezquite & 20.1 & 0.025 & Selva de galería & 2.7 & 0.003 \\
\hline $\begin{array}{l}\text { Bosque de mezquite con veg. sec. } \\
\text { arbustiva }\end{array}$ & 7.1 & 0.009 & Selva mediana caducifolia & 21.7 & 0.027 \\
\hline Bosque de oyamel & 49.0 & 0.062 & $\begin{array}{l}\text { Selva mediana caducifolia con veg. sec. } \\
\text { arbustiva }\end{array}$ & 3.1 & 0.004 \\
\hline Bosque de pino & 964.2 & 1.220 & Selva mediana subcaducifolia & 892.0 & 1.129 \\
\hline Bosque de pino con veg. sec. arbórea & 70.1 & 0.089 & $\begin{array}{l}\text { Selva mediana subcaducifolia con veg. } \\
\text { sec. arbórea }\end{array}$ & 412.4 & 0.522 \\
\hline Bosque de pino con veg. sec. arbustiva & 272.8 & 0.345 & $\begin{array}{l}\text { Selva mediana subcaducifolia con veg. } \\
\text { sec. arbustiva }\end{array}$ & 1615.2 & 2.044 \\
\hline Bosque de pino encino & 5055.5 & 6.399 & Tular & 4.9 & 0.006 \\
\hline $\begin{array}{l}\text { Bosque de pino encino con veg. sec. } \\
\text { arbórea }\end{array}$ & 943.9 & 1.195 & Vegetación de dunas costeras & 12.1 & 0.015 \\
\hline $\begin{array}{l}\text { Bosque de pino encino con veg. sec. } \\
\text { arbustiva }\end{array}$ & 1856.3 & 2.350 & Vegetación de galería & 6.0 & 0.008 \\
\hline Bosque de táscate & 122.1 & 0.155 & Vegetación halófila hidrófila & 68.7 & 0.087 \\
\hline $\begin{array}{l}\text { Bosque de táscate con veg. sec. } \\
\text { arbustiva }\end{array}$ & 106.0 & 0.134 & Vegetación halófila xerófila & 0.9 & 0.001 \\
\hline Bosque mesófilo con veg. sec. arbórea & 11.9 & 0.015 & Agrícola pecuaria forestal & 24337.0 & 30.804 \\
\hline $\begin{array}{l}\text { Bosque mesófilo con veg. sec. } \\
\text { arbustiva }\end{array}$ & 77.1 & 0.098 & Área desprovisto de vegetación & 4.3 & 0.005 \\
\hline Bosque mesófilo de montana & 339.0 & 0.429 & Asentamientos humanos & 163.1 & 0.206 \\
\hline Manglar & 27.4 & 0.035 & Cuerpo de agua & 1704.8 & 2.158 \\
\hline Manglar con veg. sec. arbustiva & 0.9 & 0.001 & Pastizal halófilo & 8.8 & 0.011 \\
\hline Matorral crasicaule & 63.5 & 0.080 & Pastizal inducido & 4608.5 & 5.833 \\
\hline $\begin{array}{l}\text { Matorral crasicaule con veg. sec. } \\
\text { arbustiva }\end{array}$ & 56.2 & 0.071 & Sin vegetación aparente & 11.5 & 0.015 \\
\hline Palmar natural & 16.0 & 0.020 & Zona urbana & 1043.7 & 1.321 \\
\hline Pastizal natural & 1359.9 & 1.721 & Total & 79005.2 & 100.0 \\
\hline
\end{tabular}

de dispersión de 1.1 kilómetros atribuida al venado cola blanca; resalta el; parche situado en la parte sur-suroeste-oeste del estado, el cual ocupa el 55.2\% de la cobertura forestal y por lo tanto, el 
mayor valor acumulado de $\operatorname{dIIC}_{k}(73.2 \%$ de 113.1), que representa el elemento de hábitat con una conectividad muy alta (Tabla II y Figura 3).

Tabla II. Rango de conectividad (importancia relativa de $k$ ), número de parches forestales, superficie y perímetro del Conjunto del Paisaje en el estado de Jalisco.

\begin{tabular}{llllll}
\hline & Conectividad & Núm. Parches & Suma de $d I I C_{k}$ & Sup. $\left(\mathbf{k m}^{2}\right)$ & Perímetro $(\mathbf{k m})$ \\
\hline \multirow{2}{*}{ IIIC $_{k}$} & Muy baja & 535 & 4.3 & 3126.5 & 7095.1 \\
& Baja & 9 & 3.0 & 1713.7 & 2192.2 \\
& Media & 2 & 7.2 & 3375.5 & 3018.5 \\
& Alta & 1 & 25.4 & 12789.2 & 10080.9 \\
& Muy alta & 1 & 73.2 & 25848.4 & 17225.4 \\
& Total & $\mathbf{5 4 8}$ & $\mathbf{1 1 3 . 1}$ & $\mathbf{4 6 8 5 3 . 3}$ & $\mathbf{3 9 6 1 2 . 2}$ \\
\hline
\end{tabular}

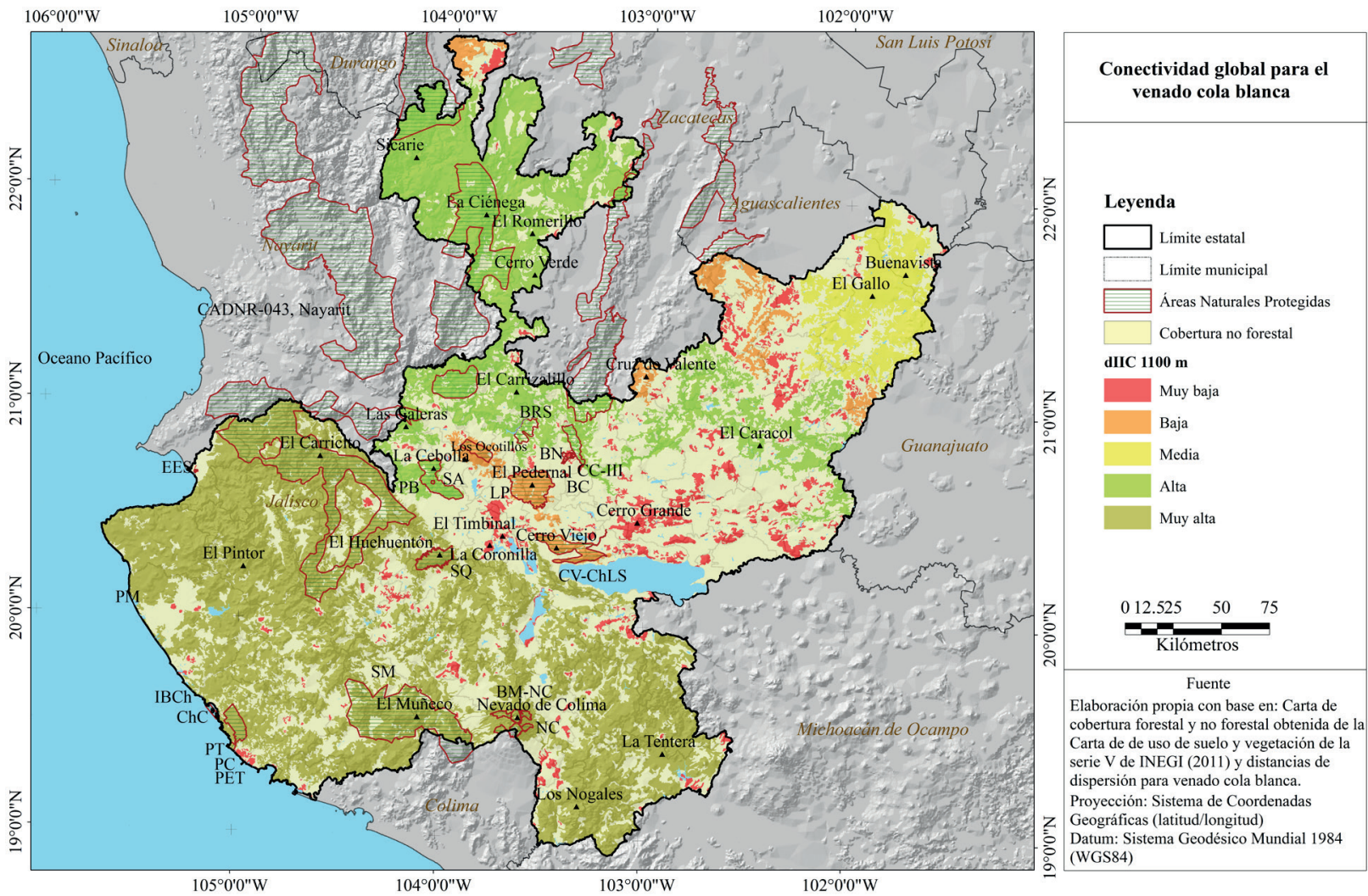

Figura 3. Conjunto del Paisaje del estado de Jalisco, El análisis de importancia relativa ${ } I I I C_{k}$ para el mantenimiento de la conectividad de parches forestales de este gráfico asume una distancia de dispersión de $1.1 \mathrm{~km}$. (Sierra de Manantlán (SM), Chamela-Cuixmala (ChC); Nevado de Colima (NC); La Primavera (LP), Sierra de Quila (SQ); Cuenca Alimentadora del Distrito Nacional de Riego 043 (CADNR-043), Islas de la Bahía de Chamela (IBCh), Playa Mismaloya (PM), Playa Teopa (PT), Playa El Tecuán (PET), Playa Cuixmala (PC), Sierra del Águila (SA), Cerro Viejo Chupinaya-Los Sabinos (CV-ChLS), Bosque Mesófilo Nevado de Colima (BM-NC), Estero El Salado (EES), Barranca del Río Santiago (BRS), Campana-Colomos III (CC-III), Bosque Los Colomos (BC), Bosque El Nixticuil (BN), Piedras Bola (PB)). 
Por el contrario, se identificaron 535 parches (6.7\% de la superficie forestal) categorizados con muy baja conectividad, por lo tanto, estos parches se consideran prioritarios para el mantenimiento de la conectividad en función de la especie; se localizan de manera dispersa en toda la extensión del estado de Jalisco, sin embargo la mayor concentración de los parches se sitúa en la zona este del estado, de manera concreta sobre los municipios de Juanacatlán, Chapala, Ixtlahuacán de Los Membrillos, Zapotlán del Rey, Tototlán y Ocotlán.

Del gráfico anterior, en acercamiento (Figura 4), destacan las áreas protegidas Sierra de Quila, Nevado de Colima, Sierra de Manantlán y Chamela Cuixmala que conectan entre sí, además de otros bosques y selvas del Sistema Montañoso Volcánico Transversal y la Sierra Madre del Sur; este complejo estructural ocupa $25,848.4 \mathrm{~km}^{2}$. Otro único parche de conectividad alta es el que se sitúa en la parte centro-norte del estado, cubre una superficie de $12,789.2 \mathrm{~km}^{2}$ y en este se encuentran las áreas protegidas estatales Sierra del Águila, Piedras Bola, varios polígonos de la Cuenca Alimentadora del Distrito Nacional de Riego 043 y la Barranca del Río Santiago.

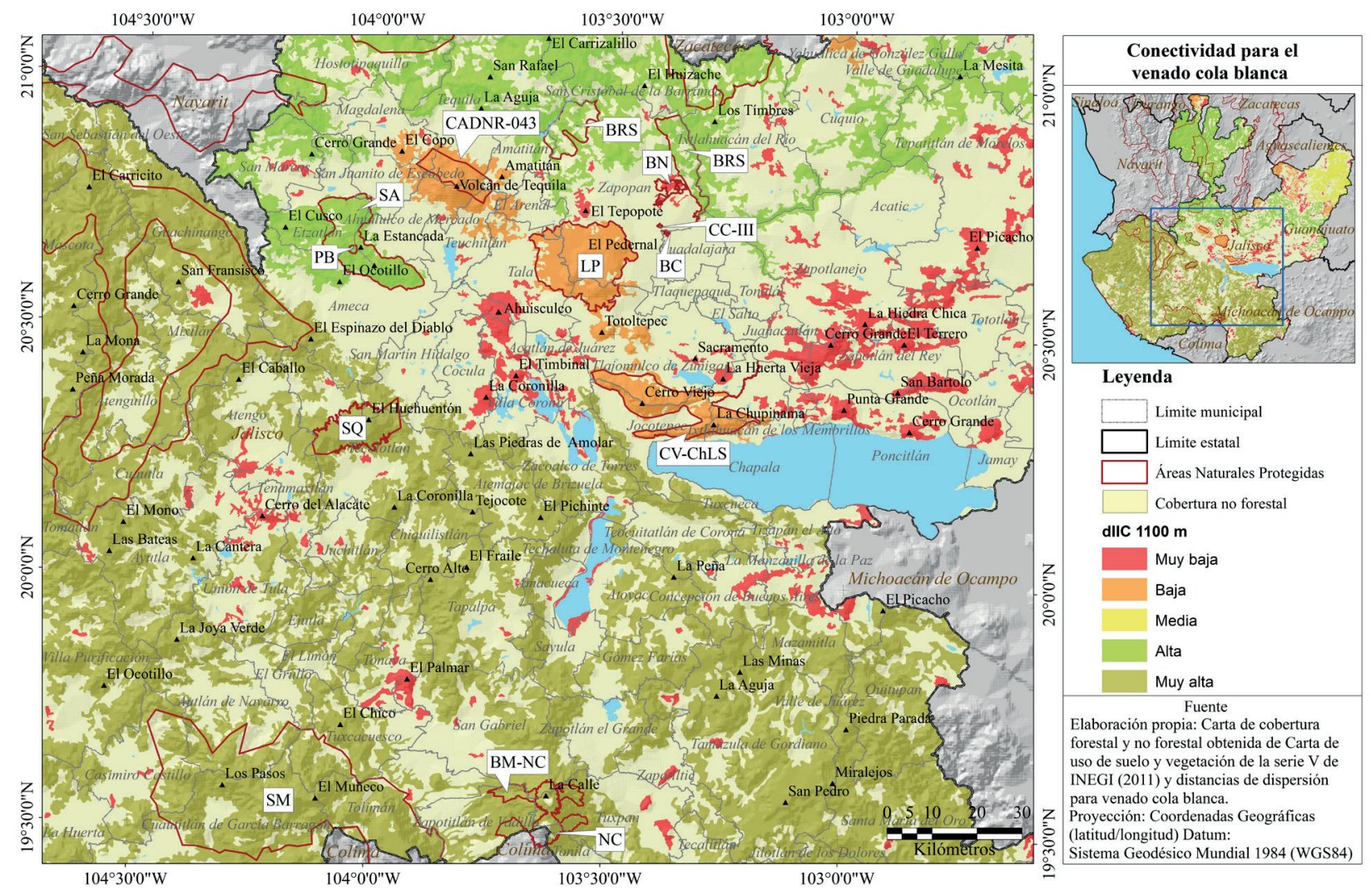

Figura 4. Acercamiento parte central del Conjunto del Paisaje del estado de Jalisco. El análisis de importancia relativa dIIC $C_{k}$ para el mantenimiento de la conectividad de parches forestales de este gráfico, asume una distancia de dispersión de $1.1 \mathrm{~km}$. (Sierra de Manantlán (SM), Nevado de Colima (NC); La Primavera (LP), Sierra de Quila (SQ); Cuenca Alimentadora del Distrito Nacional de Riego 043 (CADNR-043), Sierra del Águila (SA), Cerro Viejo Chupinaya-Los Sabinos (CV-ChLS), Bosque Mesófilo Nevado de Colima (BM-NC), Barranca del Río Santiago (BRS), Campana-Colomos III (CC-III), Bosque Los Colomos (BC), Bosque El Nixticuil (BN), Piedras Bola (PB)). 
Tres áreas de conservación se localizan en parches forestales con un rango de importancia de conectividad baja, estas son: dos polígonos de áreas protegidas federales, la CADNR-043 ubicado sobre el volcán de Tequila y La Primavera, así como una reserva estatal: el Cerro Viejo ChupinayaLos Sabinos; de considerarse que estas áreas puedan mantenerse en una red de conexión entre las áreas protegidas situadas a su alrededor con una condición de conectividad alta, los parches del CADNR-043 del volcán Tequila, LP y CV-ChLS resultan prioritarios para el mantenimiento de la conectividad, asimismo los parches forestales con muy baja conectividad que colindan por ejemplo al norte de La Primavera, el cerro El Tepopote (municipio de Zapopan), al suroeste la Sierra de Ahuisculco (municipio de Tala) y el complejo cerril del Timbinal y La Coronilla (municipio de Acatlán de Juárez); por otro lado, en el extremo noreste del Cerro Viejo Chupinaya-Los Sabinos se identifican parches forestales críticos como el complejo cerril de cerro Grande, La Hiedra Chica y El Picacho (municipios de Zapotlanejo, Zapotlán del Rey, Tototlán y Tepatitlán de Morelos) que conducen a otros espacios naturales como la Sierra de Lobos (Figura 4).

Otras áreas protegidas situadas sobre elementos de hábitat críticos en este recuadro (Figura 4) son: La Campana-Colomos III (CC-III), El Bosque Los Colomos (BC) y El Bosque El Nixticuil (BN); las dos primeras están inmersas dentro de la zona metropolitana de Guadalajara, la tercera está siendo fuertemente rodeada por complejos habitacionales en el municipio de Zapopan, lo que las limita a fungir no solo como elementos de disponibilidad de hábitat para el venado cola blanca, sino también a reducir su función como parches puente.

La Tabla III muestra en síntesis los valores y rango de importancia de los elementos de hábitat del paisaje para el mantenimiento de la conectividad y disponibilidad de hábitat del Conjunto del Paisaje por fracción; en este contexto, la fracción dIICIntra sumó el 89.8\% de 113.1 (valor total acumulado de $d I I C_{k}$ ) en sólo dos parches, esto por poseer la mayor extensión territorial dentro del Conjunto del Paisaje.

Por su amplia distribución espacial y compleja forma, tres parches reflejan la mejor conexión (muy alta y alta) que poseen con respecto a otros parches (dIICFlux), por el contrario fueron 536 parches que en este sentido, no reflejaron estar bien conectados (muy baja y baja). Sólo el 2.9 de $113.1 \%$ del dIIC $_{k^{\prime}}$ es ocupado por la fracción dIICConnector; se muestran en 531 parches clasificados como muy baja contribución como elementos conectores; el parche forestal de mayor superficie del Conjunto del Paisaje también obtuvo una clasificación baja, lo anterior puede deberse a la ubicación 
topológica del mismo (Tabla III). Los gráficos de las diferentes fracciones de dIIC $_{k}$ del Conjunto del Paisaje del estado de Jalisco se encuentran en el apartado de Anexos.

\begin{tabular}{|c|c|c|c|c|c|}
\hline & Conectividad & Núm. Parches & Suma de $d I I C$ & Sup. $\left(\mathbf{k m}^{2}\right)$ & Perímetro $(\mathrm{km})$ \\
\hline \multirow{6}{*}{ dIICIntra } & Muy baja & 538 & 0.0 & 3296.6 & 7481.7 \\
\hline & Baja & 7 & 0.1 & 1898.1 & 2132.8 \\
\hline & Media & 1 & 1.0 & 3021.0 & 2691.3 \\
\hline & Alta & 1 & 17.4 & 12789.2 & 10080.9 \\
\hline & Muy alta & 1 & 71.3 & 25848.4 & 17225.4 \\
\hline & Total & 548 & 89.8 & 46853.3 & 39612.2 \\
\hline \multirow{5}{*}{ dIICFlux } & Muy baja & 516 & 1.9 & 2142.4 & 5526.9 \\
\hline & Baja & 20 & 1.7 & 990.0 & 1584.0 \\
\hline & Media & 9 & 2.9 & 2062.3 & 2503.6 \\
\hline & Alta & 1 & 1.8 & 25848.4 & 17225.4 \\
\hline & Muy alta & 2 & 12.2 & 15810.2 & 12772.3 \\
\hline \multirow{7}{*}{ dIICConnector } & Total & 548 & 20.5 & 46853.3 & 39612.2 \\
\hline & Muy baja & 531 & 1.2 & 6970.6 & 10479.7 \\
\hline & Baja & 10 & 0.3 & 26437.2 & 18072.3 \\
\hline & Media & 4 & 0.4 & 431.5 & 556.9 \\
\hline & Alta & 2 & 0.5 & 224.8 & 422.3 \\
\hline & Muy alta & 1 & 0.6 & 12789.2 & 10080.9 \\
\hline & Total & 548 & 2.9 & 46853.3 & 39612.2 \\
\hline
\end{tabular}

Para comparar las tendencias de cambio de los parches forestales entre las tres fracciones del dIIC para el venado cola blanca, se muestran los valores relativos de importancia de los parches en las Figuras 5, 6 y 7 como resultado gráfico del análisis de conectividad. La respuesta de los parches para la fracción dIICintra fue similar a la obtenida para $d I I C_{k^{\prime}}$ por lo que se puede especificar que los parches forestales que contienen las veinte áreas protegidas reflejaron los mismos valores y categorías de importancia (Figura 5).

La Sierra del Águila (SA), Piedras Bola (PB), algunos polígonos de la Cuenca Alimentadora del Distrito Nacional de Riego 043 (CADNR-043) y la Barranca del Río Santiago (BRS) se sitúan sobre un mismo parche forestal con la característica de estar bien conectado (muy alta); la misma tendencia ocurre para las Sierras de Quila (SQ), Manantlán (SM), el Nevado de Colima (NC) y el Bosque Mesófilo Nevado de Colima (BM-NC). La Primavera, el Cerro Viejo Chupinaya-Los Sabinos y un polígono de la CADNR-043 (donde se sitúan el volcán Tequila) reflejaron valores 
medios de conectividad, es decir, mantienen una posición intermedia de conexión con el resto del hábitat existente en el paisaje (Figura 6).

Por último la fracción dIICConnector se muestra en la Figura 7, donde el parche forestal de mayor extensión del Conjunto del Paisaje y, que alberga las áreas protegidas Sierras de Quila (SQ), Manantlán (SM), el Nevado de Colima (NC), el Bosque Mesófilo Nevado de Colima (BM-NC), Chamela Cuixmala (ChC) e incluso un polígono de la CADNR-043 se caracteriza con un rango bajo como parche conector o parche puente. Por el contrario los parches de hábitat que contribuyen de manera muy alta como elementos conectores entre el resto de los parches de hábitat son aquellas donde se sitúan las áreas protegidas Sierra del Águila (SA), Piedras Bola (PB), algunos polígonos de la CADNR-043, la Barranca del Río Santiago (BRS) y La Primavera (LP).

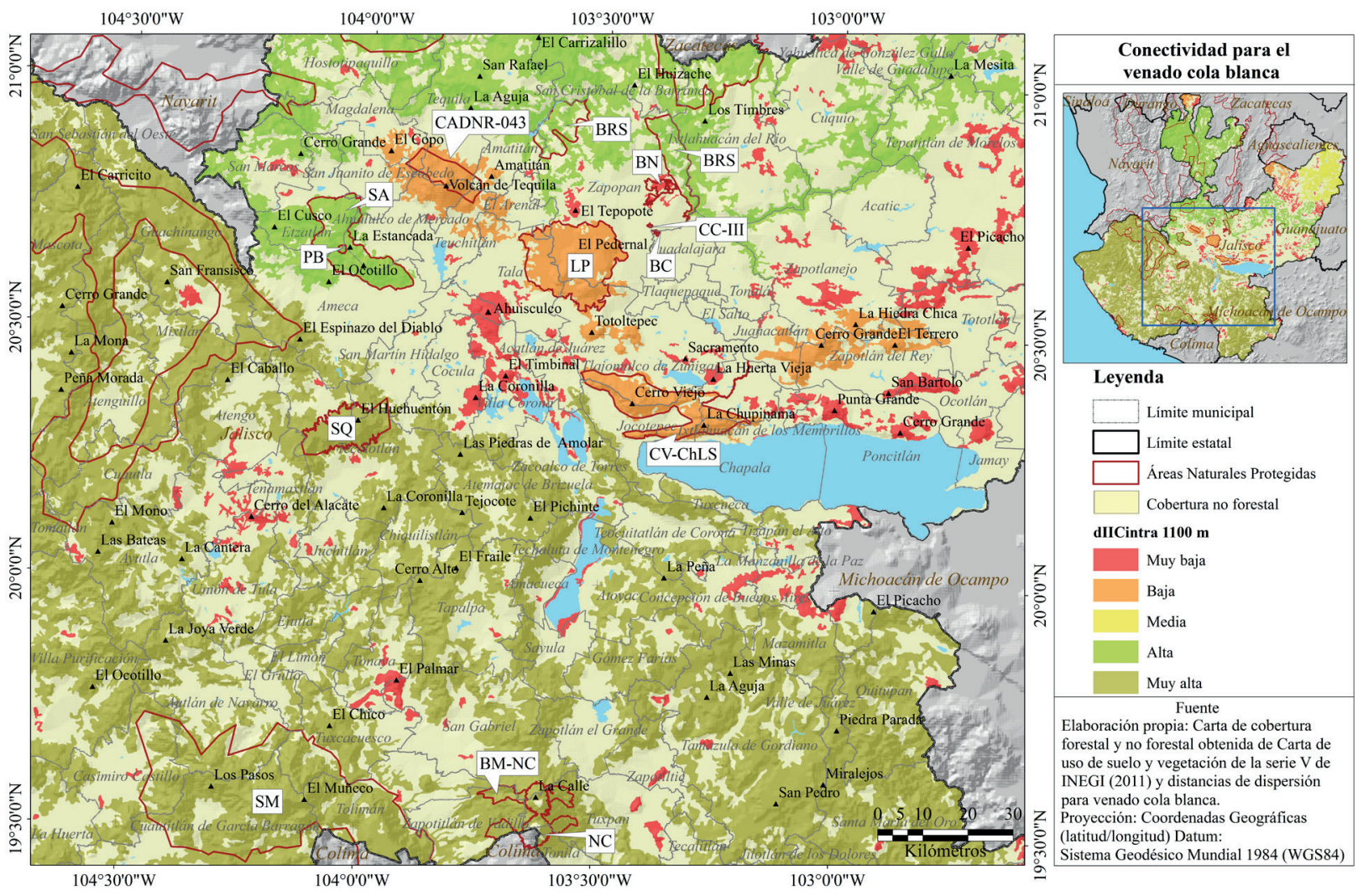

Figura 5. Acercamiento de la parte central del Conjunto del Paisaje del estado de Jalisco y conectividad de los parches en término de contribución de área o hábitat disponible (dIICIntra $)_{k}$; este gráfico asume una distancia de dispersión de $1.1 \mathrm{~km}$. (Sierra de Manantlán (SM), Nevado de Colima (NC); La Primavera (LP), Sierra de Quila (SQ); Cuenca Alimentadora del Distrito Nacional de Riego 043 (CADNR-043), Sierra del Águila (SA), Cerro Viejo Chupinaya-Los Sabinos (CV-ChLS), Bosque Mesófilo Nevado de Colima (BM-NC), Barranca del Río Santiago (BRS), Campana-Colomos III (CC-III), Bosque Los Colomos (BC), Bosque El Nixticuil (BN), Piedras Bola $(\mathrm{PB}))$. 
Las fracciones en las que se divide el $d I I C_{k^{\prime}}$ influye en la función de cada uno de los parches o elementos de hábitat, ya que estos no sólo sirven como sitios de refugio, de alimento y de reproducción, sino también producen o reciben flujos de dispersión a otros o desde otros parches de hábitat; también funcionan como puntos de paso, es decir, la especie solo usa este parche con un fin transitorio. De acuerdo a De La Cruz y Maestre (2013), la contribución de cada una de estas fracciones a la disponibilidad global del hábitat y la conectividad va a depender de los atributos de las especies, por ejemplo, si son especies con baja movilidad (habito hogareño pequeño) o con grandes capacidades de dispersión. Por su lado, Saura et al. (2011) señalan que las fracciones del IIC, permiten obtener una visión adicional y directa sobre la disponibilidad de hábitat y la funcionalidad de cada uno de los parches para la dispersión animal en el paisaje, asimismo se pueden identificar aquellos parches críticos que sustentan la conectividad en el paisaje y como, en un escenario negativo, su disminución o desaparición, puede afectar los requerimientos o necesidades del animal.

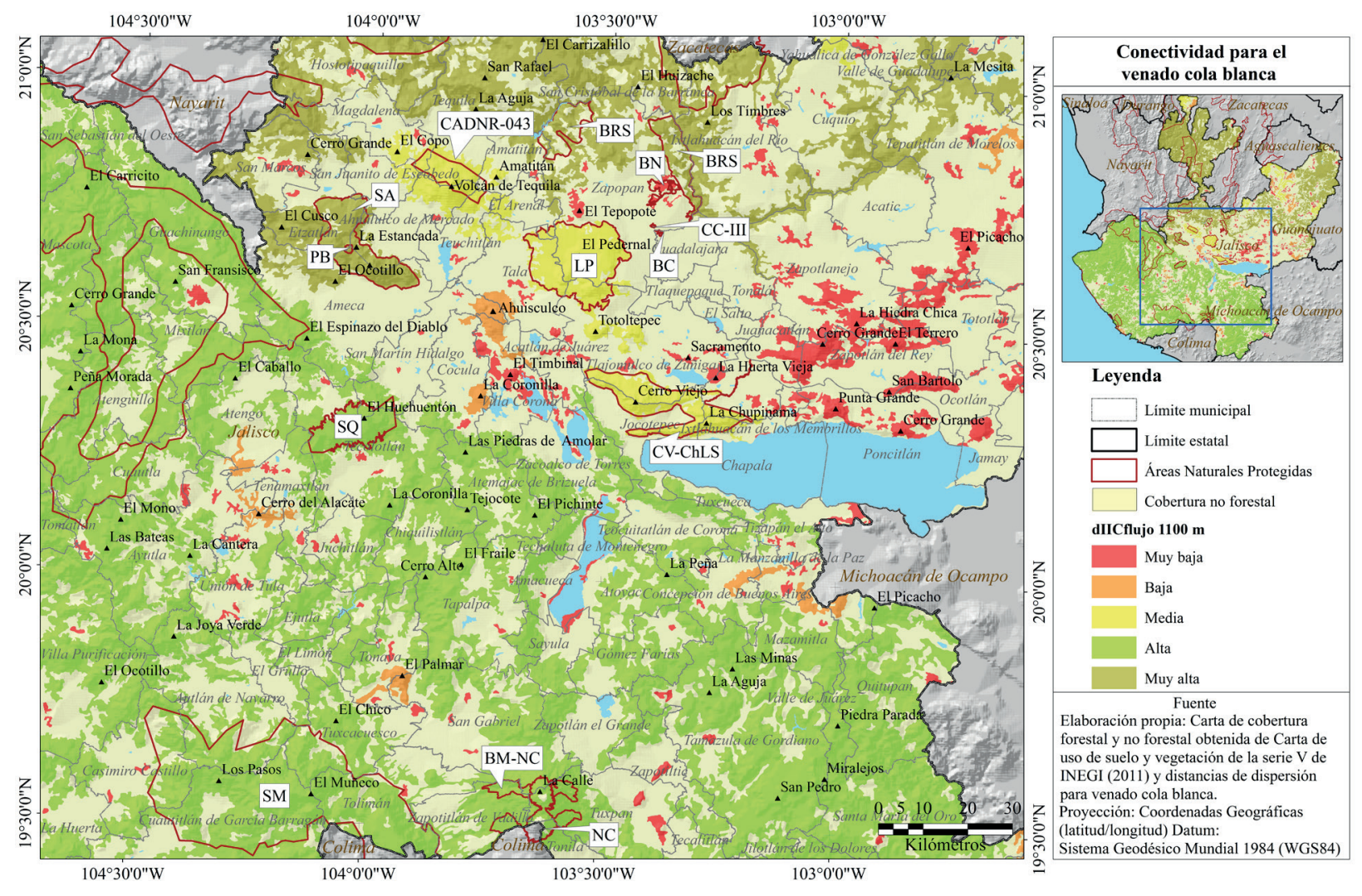

Figura 6. Acercamiento de la parte central del Conjunto del Paisaje del estado de Jalisco y conectividad de los parches con otros elementos del paisaje (dIICFlux $x_{k}$ ); este gráfico asume una distancia de dispersión de $1.1 \mathrm{~km}$. (Sierra de Manantlán (SM), Nevado de Colima (NC); La Primavera (LP), Sierra de Quila (SQ); Cuenca Alimentadora del Distrito Nacional de Riego 043 (CADNR-043), Sierra del Águila (SA), Cerro Viejo Chupinaya-Los Sabinos (CV-ChLS), Bosque Mesófilo Nevado de Colima (BM-NC), Barranca del Río Santiago (BRS), Campana-Colomos III (CC-III), Bosque Los Colomos (BC), Bosque El Nixticuil (BN), Piedras Bola (PB)). 
En síntesis, los diferentes parches pueden tener diferentes funciones dependiendo de su posición topológica (ubicación, proximidad, conectividad) y su característica intrínseca del hábitat (calidad y disponibilidad), en este contexto, la Tabla IV muestra la categoría de importancia para el mantenimiento de la conectividad de los parches de cobertura forestal que de manera directa sitúa a cada una de las áreas protegidas por cada fracción de $d I I C_{k^{\prime}}$ cabe resaltar que la Cuenca Alimentadora del Distrito Nacional de Riego 043 se compone de doce polígonos disgregados sobre los estados de Durango, Nayarit, Jalisco, Zacatecas y Aguascalientes, sin embargo el rango de importancia de la conectividad para esta área protegida a los cinco polígonos localizados en el estado de Jalisco, los cuales se categorizan con valores altos y muy altos de conectividad de manera general y en las tres fracciones de $d I I C_{k}$ sólo el polígono que se sitúa en el parche forestal del volcán Tequila presentó valores bajo en IIIC $_{k}$ y dIICIntra, medio en dIICFlux y muy bajo en dIICConnector (ver Tabla IV).

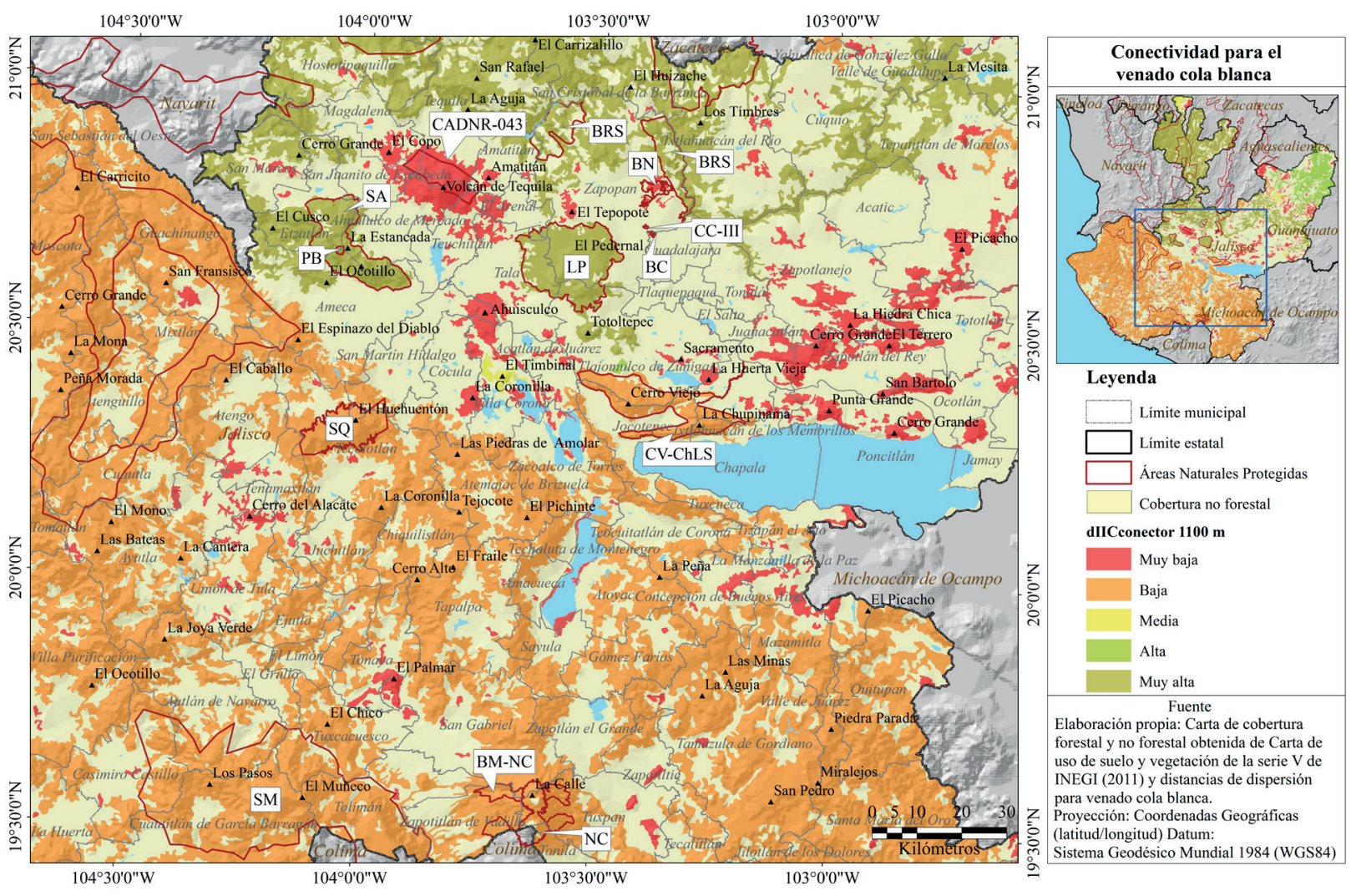

Figura 7. Acercamiento parte central del Conjunto del Paisaje del estado de Jalisco caracterizando la contribución de cada parche como elemento conector entre el resto de los parches (dIICConnector $\left.{ }_{k}\right)$; este gráfico asume una distancia de dispersión de $1.1 \mathrm{~km}$. (Sierra de Manantlán (SM), Nevado de Colima (NC); La Primavera (LP), Sierra de Quila (SQ); Cuenca Alimentadora del Distrito Nacional de Riego 043 (CADNR-043), Sierra del Águila (SA), Cerro Viejo Chupinaya-Los Sabinos (CV-ChLS), Bosque Mesófilo Nevado de Colima (BM-NC), Barranca del Río Santiago (BRS), Campana-Colomos III (CC-III), Bosque Los Colomos (BC), Bosque El Nixticuil (BN), Piedras Bola (PB)). 
El volcán Tequila de 2,900 metros, forma parte de la región agavera de los municipios de El Arenal, Amatitán, Tequila y Teuchitlán, motivo por el cual, le convierte en uno de los paisajes culturales más importantes de México; sin embargo, en términos de conectividad este parche forestal es vulnerable y puede convertirse en un parche de vegetación remanente y disfuncional como corredor de hábitat o parche de paso, debido a la vecindad de parcelas de uso agrícola intensivo (agave y caña de azúcar) que le rodean, así como a la paulatina modificación territorial en su entorno (infraestructura). De acuerdo a la CONANP (2017), los Santuarios son zonas caracterizadas con una considerable riqueza de flora o fauna o por la presencia de especies o hábitat de distribución restringida; para Jalisco a excepción de las Islas de la Bahía de Chamela, el resto de los Santuarios son playas que regularmente se encuentran desprovistas de vegetación o bien, en algunas de estas se desarrolla la vegetación de dunas costeras. Para este estudio, los rangos de importancia para el mantenimiento de la conectividad sobre los Santuarios (playas), se categorizaron por el valor atribuido del parche forestal inmediato a cada uno de los Santuarios (ver Tabla IV).

La conectividad forestal se traduce en la conexión funcional del hábitat en el territorio, la cual puede lograrse mediante una continuidad estructural sin frontera o bien, depender de la habilidad de la especie para dispersarse; en este contexto, Bennett (1998) sugiere que una manera de contrarrestar los problemas que surgen de la fragmentación será: ampliar el área de los hábitats protegidos, maximizar la calidad los elementos de hábitat existentes mediante buenas prácticas de manejo y minimizar mediante planes de ordenamiento territorial los impactos por uso de tierras circundantes; por último, fomentar mecanismos que coadyuven a la conectividad y contrarresten el efecto de aislamiento de los elementos de hábitat. Entre otras observaciones, Saura y Rubio (2010) señalan, que dentro de las estrategias de conectividad es importante reconocer que algunos parches dispersos pueden servir como sitios de paso, siendo esto más deseable, que la desaparición total de las coberturas originales; estos sitios de paso son utilizados para llegar a otros parches de hábitat, por lo cual también se debe considerar que la distancia entre parches y sitios de paso no sean tan largos, ya que es probable que no sea utilizada por la fauna silvestre.

Al respecto, Dramstad et al. (2005) aseguran que la pérdida de un pequeño parche, intermedio entre otros parches, cuya función es ser punto de apoyo para el movimiento de las especies, inhibe el movimiento y por lo tanto, incrementa el aislamiento entre parches; además mencionan que un 
Tabla IV. Rango de importancia para el mantenimiento de la conectividad por fracción para el venado cola blanca, que atribuye cada parche forestal que ocupan las áreas naturales protegidas en el estado de Jalisco. Área de Protección de Flora y Fauna (APFF), Área de Protección de Recursos Naturales (APRN), Parque Nacional (PN), Reserva de la Biosfera (RB), Santuario (S), Área de Protección Hidrológica (APH, Parque Estatal 8PE), Zona de Conservación Ecológica (ZCE), Formación Natural (FN).

\begin{tabular}{|c|c|c|c|c|c|c|}
\hline No. & Categoría & Nombre & $d I I C_{k}$ & $\begin{array}{l}d I I C \\
\text { Intra }\end{array}$ & $\begin{array}{l}d I I C \\
\text { Flux }\end{array}$ & $\begin{array}{l}\text { dIIC } \\
\text { Connector }\end{array}$ \\
\hline 1 & APFF & La Primavera & Baja & Baja & Media & Muy alta \\
\hline 2 & APFF & Sierra de Quila & Muy alta & Muy alta & Alta & Baja \\
\hline 3 & APRN & CADNR 043 (volcán Tequila) & Baja & Baja & Media & Muy baja \\
\hline 4 & $\mathrm{PN}$ & Nevado de Colima & Muy alta & Muy alta & Alta & Baja \\
\hline 5 & $\mathrm{RB}$ & Sierra de Manantlán & Muy alta & Muy alta & Alta & Baja \\
\hline 6 & $\mathrm{RB}$ & Chamela Cuixmala & Muy alta & Muy alta & Alta & Baja \\
\hline 7 & S & Islas de la Bahía de Chamela & Muy baja & Muy baja & Muy baja & Muy baja \\
\hline 8 & S & Playa Mismaloya & Muy alta & Muy alta & Alta & Baja \\
\hline 9 & $S$ & Playa Teopa & Muy alta & Muy alta & Alta & Baja \\
\hline 10 & S & Playa El Tecuan & Muy baja & Muy baja & Baja & Muy baja \\
\hline 11 & S & Playa Cuixmala & Muy alta & Muy alta & Alta & Baja \\
\hline 12 & $\mathrm{APH}$ & Sierra del Águila & Alta & Alta & Muy alta & Muy alta \\
\hline 13 & $\mathrm{APH}$ & Cerro Viejo Chupinaya Los Sabinos & Baja & Baja & Media & Baja \\
\hline 14 & PE & Bosque Mesófilo Nevado de Colima & Muy alta & Muy alta & Alta & Baja \\
\hline 15 & $\mathrm{ZCE}$ & Estero El Salado & Muy baja & Muy baja & Muy baja & Muy baja \\
\hline 16 & $\mathrm{APH}$ & Barranca del Río Santiago & Alta & Alta & Muy alta & Muy alta \\
\hline 17 & $\mathrm{APH}$ & La Campana Colomos III & Muy baja & Muy baja & Muy baja & Muy baja \\
\hline 18 & $\mathrm{APH}$ & Bosque Los Colomos & Muy baja & Muy baja & Muy baja & Muy baja \\
\hline 19 & $\mathrm{APH}$ & Bosque El Nixticuil & Muy baja & Muy baja & Muy baja & Muy baja \\
\hline 20 & $\mathrm{FN}$ & Piedras Bola & Alta & Alta & Muy alta & Muy alta \\
\hline
\end{tabular}

parche pequeño que está conectado a la red de corredores tiende a tener un número ligeramente mayor de especies y una menor tasa de extinción que un parche del mismo tamaño que este separado de la red; en este mismo sentido, De La Cruz y Maestre (2013) sugieren que la continuidad física (estructural) del hábitat garantiza la conectividad para las especies menos móviles y más 
sensibles a los efectos de la fragmentación como algunos roedores (por ejemplo: Sigmodon alleni y Neotomodon alstoni), la tuza de Colima (Cratogeomys gymnurus), el tlacuache (Didelphis virginiana) o el conejo (Syvilagus floridanus) (Correa et al., 2017; Sutherland et al., 2000) y, una vez garantizada la posibilidad de dispersión de estas, se asume que también quedará asegurada para el resto de especies con mayores capacidades de movimiento; como el coyote (Canis latrans), la zorra gris (Urocyon cinereoargenteus), el lince (Lynx rufus) o el puma (Puma concolor).

\section{Conclusiones}

El Conjunto del Paisaje del estado de Jalisco que albergaría el hábitat forestal para el venado cola blanca presentó de manera general una alta conectividad de acuerdo al índice de importancia relativa $\left({ } I I C_{k}\right)$ en cuarenta y cinco municipios del norte y noreste del estado y, muy alta en sesenta y dos municipios localizados al sur, suroeste y oeste.

Las regiones centro, este y noreste del estado (cincuenta municipios) presentan una falta de cobertura forestal continua y por consiguiente un mayor número de parches críticos, originado principalmente por la expansión agrícola, ganadera y urbana, lo que dificultará la capacidad dispersiva y de supervivencia de esta especie.

El IIC aunado a un SIG, proporcionó un panorama regional actual de la condición funcional del hábitat forestal para el venado cola blanca aún fuera de las áreas protegidas; la Cuenca Alimentadora del Distrito Nacional de Riego 043, Sierra de Quila, Nevado de Colima, Bosque Mesófilo Nevado de Colima, Sierra de Manantlán y Chamela Cuixmala forman parte de un complejo forestal importante por la disponibilidad de hábitat, estar conexo y contribuir al flujo dispersivo no solo del venado cola blanca, sino de diversas especies hacia otros elementos del paisaje del occidente de México.

Con el propósito de mantener la conectividad del hábitat a mediano y largo plazo y asegurar el éxito dispersivo de la especie hacia otros espacios naturales, deben considerarse como prioritarios aquellos parches categorizados con valor muy bajo, bajo y medio del $d I I C_{k}$ representados en las fracciones intra, flux y connector.

Se recomienda a nivel municipal, mantener la conectividad entre parches críticos a través de acciones tendientes a la planificación del uso del suelo como son: la ampliación de hábitats protegidos, maximizar la calidad de los hábitats existentes, minimizar los impactos de usos de tierras y fomentar la conectividad de hábitats para contrarrestar los efectos del aislamiento. 


\section{Literatura citada}

Bennet, A. F. 1998. Linkages in the Landscape: The Role of Corridors and Connectivity in Wildlife Conservation. IUCN. Gland, Suiza and Cambridge, UK. 254 pp.

Bello, G. J., S. Gallina, y M. Equihua. 2004. Distancias de desplazamiento del venado cola blanca y su relación con factores ambientales en el Noreste de México. Memorias del VI Congreso internacional sobre manejo de fauna silvestre en la Amazonia y Latinoamérica. 5-10 septiembre 2004. Iquitos, Perú. 146-151 pp. Consultado: 10-11-2016. En: http:/ / manejofaunasilvestre.org/ Congresos/Iquitos-2004/Ponencias.aspx.

Ceballos, G. y G. Oliva. (coord.). 2005. Los mamíferos silvestres de México. Fondo de Cultura Económica. México, D. F. 986 pp.

Comisión Nacional de Áreas Naturales Protegidas (CONANP). 2017. Áreas Naturales Protegidas. Consultado: 10-03-2017. En: http://www.conanp.gob.mx/regionales/

Correa-Ayram, C. A., M. E. Mendoza, A. Etter y D. R. Pérez-Salicrup. 2017. Anthropogenic impact on habitat connectivity: A multidimensional human footprint index evaluated in a highly biodiverse landscape of Mexico. Ecological Indicators 72 (2017): 895-909.

Chávez, G. J. M. 2012. Los significados del venado sol en la cosmovisión Maya: Un atisbo a la mitología y la historia oral mayance. Editorial Académica Española. México, D. F. 220 pp.

Chuvieco, E. 2010. Teledetección ambiental - La observación de la Tierra desde el espacio. Editorial Ariel. Barcelona, España. 590 pp.

De la Cruz, M. y F. T. Maestre. (eds.). 2013. Avances en el análisis espacial de datos ecológicos: aspectos metodológicos y aplicados. ECESPA-Asociación Española de Ecología Terrestre. Móstoles, Madrid, España. 355 pp.

Dirección General de Desarrollo Municipal (DGDM). 2017. Municipios de Jalisco. Secretaría General de Gobierno. Estado de Jalisco. Consultado: 10-10-2016. En: https://www.jalisco.gob.mx/ jalisco/municipios

Diario Oficial de la Federación (DOF). 2010. Norma Oficial Mexicana NOM-059-SEMARNAT-2010. Protección ambiental-especies nativas de México de flora y fauna silvestres-categorías de riesgo y especificaciones para su inclusión, exclusión o cambio-lista de especies en riesgo. Consultado: 02-01-2015. En: http:/ / dof.gob.mx/nota_detalle.php?codigo=5173091\&fecha=30/12/2010 
Dramstad, E. W., J. D. Olson, y R. T. T. Forman. 2005. Principios de ecología del paisaje en arquitectura del paisaje y planificación territorial. Fundación Conde del Valle de Salazar. España. 95 pp.

Fulbright, T. E. y J. A. Ortega-Santos. 2007. Ecología y manejo de venado cola blanca. Texas A y M University Press.USA. 265 pp.

Hernández, A. R. 2014. Variabilidad genética y relación filogeográfia de tres subespecies de venado cola blanca (Odocoileus virginianus) en la región centro-norte de México. Tesis de Maestría. Colegio de Posgraduados - Campus Montecillo. México. 67 pp.

Instituto Nacional de Estadística Geografía e Informática (INEGI). 2013. Conjunto de datos vectoriales de uso del suelo y vegetación, escala 1:250 000, serie V (continuo nacional). México (capa unión). Consultado: 24-06-2014. En: http://www.inegi.org.mx/geo/contenidos/recnat/usosuelo/ Default.aspx

Martínez-Alandi, C., M. Múgica-de la Guerra, C. Castell-Puig, y J. V. Lucio-Fernández. 2009. Conectividad ecológica y áreas protegidas. Herramientas y casos prácticos. Monografía: 02. Ed. FUNGOBE. EUROPARC-España. Madrid. 86 pp.

Medina-Torres, S. M., E. García-Moya, M. Márquez-Olivas, A. Romero-Manzanares, H. VaqueraHuerta, y M. Martínez-Menes. 2015. Relación hábitat-densidad de Odocoileus virginianus couesi (Coues y Yarrow, 1875) en la Sierra del Laurel, Aguascalientes. Revista Mexicana de Ciencias Forestales 6 (32): 17-36.

Ontiveros, J. C. 2012. Evaluación del crecimiento de cervatos cola blanca Texanos (Odocoileus virginianus texanus), alimentados con diferentes fórmulas lácteas. Tesis de Maestría. Universidad Autónoma de Nuevo León. México. 113 pp.

Ortiz-Martínez, T., S. Gallina, M. Briones-Salas, y G. González. 2006. Densidad poblacional y caracterización del hábitat del venado cola blanca (Odocoileus virginianus oaxacensis, Golman y Kellog, 1940) en un bosque templado de la Sierra Norte de Oaxaca, México. Acta Zoológica Mexicana 21 (3): 65-78.

Pascual-Hortal, L. y S. Saura. 2006. Comparison and development of new graph-based landscape connectivity indices: towards the priorization of habitat patches and corridors for conservation. Landscape Ecology (21): 959-967.

Saura, S., C. Estreguil, C. Mouton y M. Rodríguez-Freire. 2011. Network analysis to assess landscape connectivity trends: Application to European forest (1990-2000). Ecological Indicators 11 (2): 407416. 
Saura, S. y J. Torné. 2012. Conefor 2.6. User manual (April 2012). Universidad Politécnica de Madrid. Consultado: 12-12-2014. En: http://www.conefor.org Saura, S. y L. Rubio. 2010. A common currency for the different ways in which patches and links can contribute to habitat availability and connectivity in the landscape. Ecography 33 (3): 523-537.

Secretaria de Medio Ambiente y Recursos Naturales (SEMARNAT). 2014. Inventario estatal forestal y de suelos - Jalisco 2013. Tomo 14. - CONAFOR. México, D.F. 184 pp.

Secretaria de Medio Ambiente y Recursos Naturales (SEMARNAT). 2014b. Plan de manejo tipo para la conservación y aprovechamiento sustentable del venado cola blanca (Odocoileus virginianus) en climas templados y tropicales de México extensiva y cría en cautiverio. Consultado: 0203-2016. En: http://www.semarnat.gob.mx/archivosanteriores/temas/gestionambiental/ vidasilvestre/Documents/Planes\%20de\%20Manejo/Plan\%20de\%20Manejo\%20Tipo\% 20 de\%20Venado\%20Cola\%20Blanca\%20en\%20Zonas\%20Templadas\%20y\%20Tropicales.pdf

Sutherland, G. D., A.S. Harestad, K. Price y K. P. Lertzman. 2000. Scaling of natal dispersal distances in terrestrial birds and mammals. Conservation Ecology 4 (1): 16.

Villavicencio-Garcia, R., S. Saura, A. L. Santiago-Pérez y A. Chávez-Hernández. 2009. La conectividad forestal de las áreas protegidas del estado de Jalisco con otros ambientes naturales. Scientia-CUCBA 11 (1-2): 43-50.

Weber, M. 2014. Temazates y venados cola blanca tropicales. pp. 421-452. En: Valdez, R. y J. A. OrtegaSánchez. (eds.). Ecología y manejo de fauna silvestre en México. Colegio de Posgraduados y Biblioteca Básica de Agricultura. México. 523 pp.

Zepeda, K. 2013. Áreas Naturales Protegidas. Secretaria de Medio Ambiente y Desarrollo Territorial (SEMADET). Consultado: 12-08-2016. En: http://semadet.jalisco.gob.mx/medio-ambiente/ biodiversidad/areas-naturales-protegidas/102

\section{Cita}

Villavicencio García R.*, R. Ávila Coria, S. Guerrero Vázquez, A. L. Santiago Pérez y E. Treviño Garza. 2017. Conectividad del hábitat forestal de las áreas protegidas para el venado cola blanca (Odocoileus virginianus) en el estado de Jalisco, México. Áreas Naturales Protegidas Scripta, 2017. Vol. 3 (2): 9-31. https:/ / doi.org/10.18242/anpscripta.2017.03.03.02.0001 
Sometido: 25 de Mayo de 2017

Revisado: 18 de Julio de 2017

Aceptado: 27 de Agosto de 2017

Editor asociado: Dr. Manuel Pinkus Rendón

Idioma Inglés Abstract: Ms.C. Diana Dorantes

Diseño gráfico editorial: Lic. Gerardo Hernández

Anexo

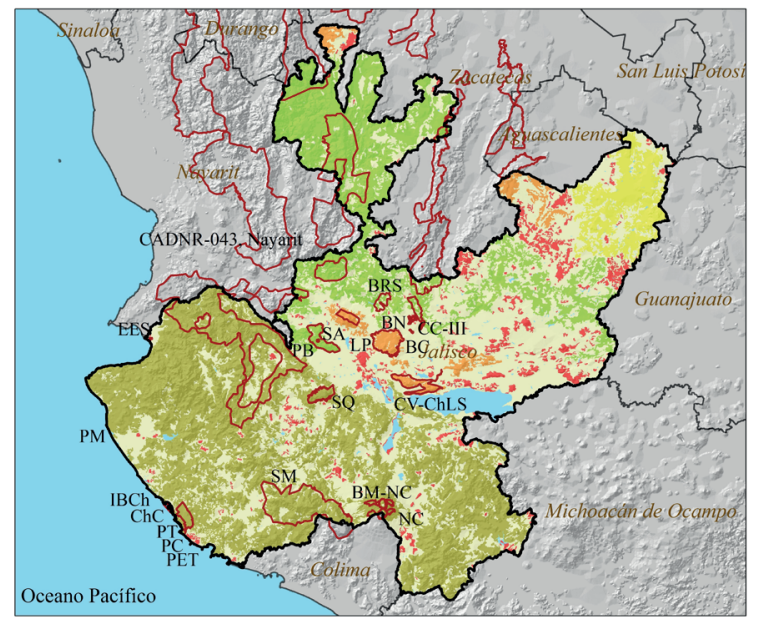

a)
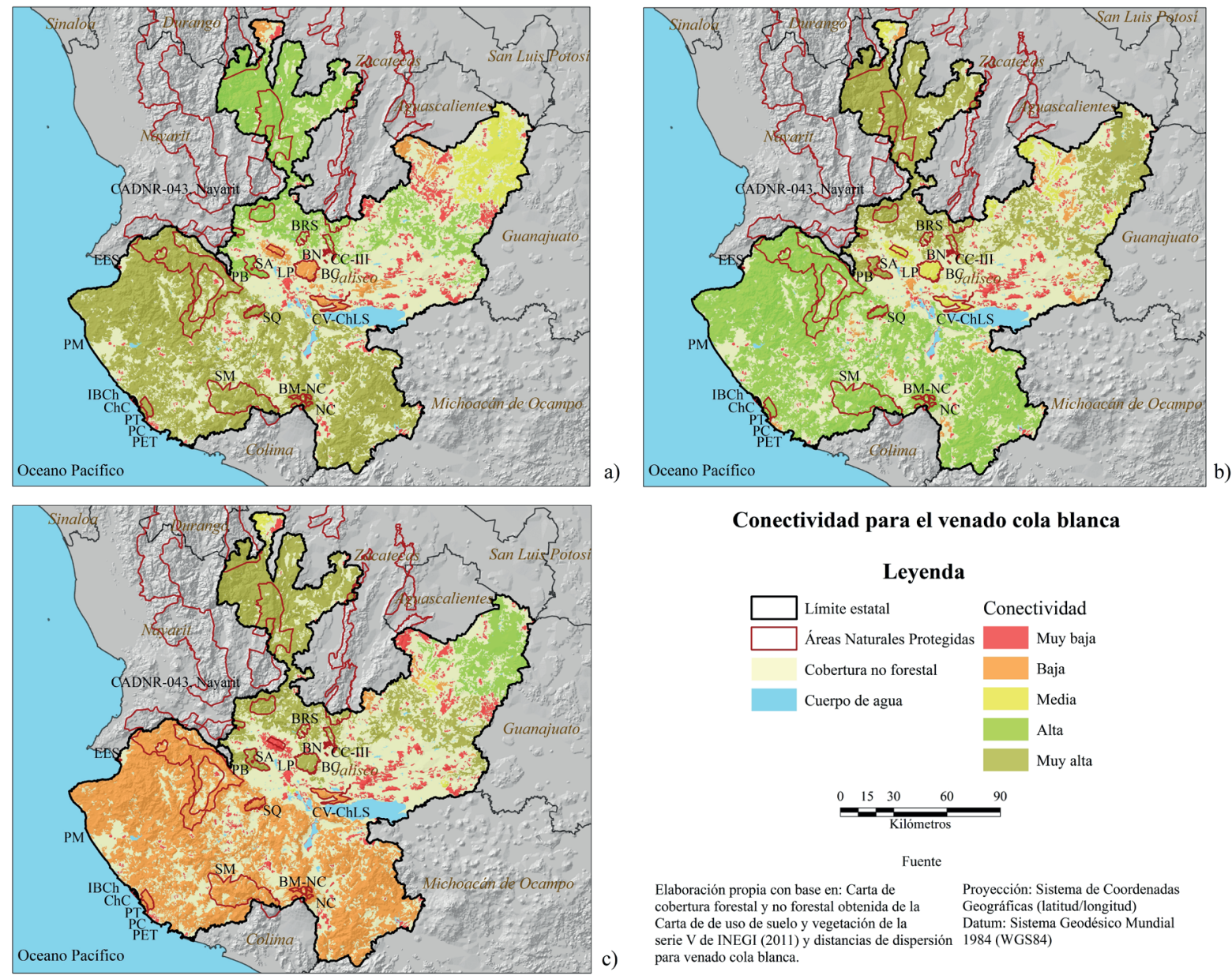

Figura 8. Conjunto del Paisaje del estado de Jalisco y conectividad de los parches en términos de: (a) la disponibilidad de hábitat (dIICIntra $_{k}$ ); (b) la conexión de los parches con otros elementos del paisaje (dIICFlux $x_{k}$ ) y (c) la contribución de cada parche como elemento conector entre el resto de los parches (dIICConnector ${ }_{k}$ ). Los gráficos asumen una distancia de dispersión de $1.1 \mathrm{~km}$. (Sierra de Manantlán (SM), Chamela-Cuixmala (ChC); Nevado de Colima (NC); La Primavera (LP), Sierra de Quila (SQ); Cuenca Alimentadora del Distrito Nacional de Riego 043 (CADNR-043), Islas de la Bahía de Chamela (IBCh), Playa Mismaloya (PM), Playa Teopa (PT), Playa El Tecuán (PET), Playa Cuixmala (PC), Sierra del Águila (SA), Cerro Viejo Chupinaya-Los Sabinos (CV-ChLS), Bosque Mesófilo Nevado de Colima (BM-NC), Estero El Salado (EES), Barranca del Río Santiago (BRS), Campana-Colomos III (CC-III), Bosque Los Colomos (BC), Bosque El Nixticuil (BN), Piedras Bola (PB)). 